ARTICLE

Received 21 Dec 2015 | Accepted 20 May 2016 | Published 21 Jun 2016

DOI: $10.1038 /$ ncomms12011

OPEN

\title{
Thermoelectric materials by using two-dimensional materials with negative correlation between electrical and thermal conductivity
}

Myoung-Jae Lee ${ }^{1,2, \star}$, Ji-Hoon Ahn ${ }^{2,3, \star}$, Ji Ho Sung ${ }^{2,4}$, Hoseok Heo ${ }^{2,4}$, Seong Gi Jeon ${ }^{5,6}$, Woo Lee ${ }^{6}$, Jae Yong Song ${ }^{6}, \mathrm{Ki}-\mathrm{Ha}$ Hong ${ }^{7}$, Byeongdae Choi ${ }^{1}$, Sung-Hoon Lee ${ }^{2} \&$ Moon-Ho Jo ${ }^{2,4,8}$

In general, in thermoelectric materials the electrical conductivity $\sigma$ and thermal conductivity $\kappa$ are related and thus cannot be controlled independently. Previously, to maximize the thermoelectric figure of merit in state-of-the-art materials, differences in relative scaling between $\sigma$ and $\kappa$ as dimensions are reduced to approach the nanoscale were utilized. Here we present an approach to thermoelectric materials using tin disulfide, $\mathrm{SnS}_{2}$, nanosheets that demonstrated a negative correlation between $\sigma$ and $\kappa$. In other words, as the thickness of $\mathrm{SnS}_{2}$ decreased, $\sigma$ increased whereas $\kappa$ decreased. This approach leads to a thermoelectric figure of merit increase to 0.13 at $300 \mathrm{~K}$, a factor $\sim 1,000$ times greater than previously reported bulk single-crystal $\mathrm{SnS}_{2}$. The Seebeck coefficient obtained for our two-dimensional $\mathrm{SnS}_{2}$ nanosheets was $34.7 \mathrm{mV} \mathrm{K}^{-1}$ for 16 -nm-thick samples at $300 \mathrm{~K}$.

\footnotetext{
${ }^{1}$ Division of Nano and Energy Convergence Research, Daegu Gyeongbuk Institute of Science and Technology (DGIST), 333, Techno Jungang Daero, Hyeonpung-Myeon, Dalseong-Gun, Daegu 42988, Korea. ${ }^{2}$ Center for Artificial Low Dimensional Electronic Systems, Institute for Basic Science (IBS), Pohang University of Science and Technology (POSTECH), Pohang 790-784, Korea. ${ }^{3}$ Department of Electronic Material Engineering, Korea Maritime and Ocean University, Busan 49112, Korea. ${ }^{4}$ Division of Advanced Materials Science, Pohang University of Science and Technology (POSTECH), Pohang 790-784, Korea. ${ }^{5}$ Korea Advanced Institute of Science and Technology (KAIST), Daejeon 305-701, Korea. ${ }^{6}$ Korea Research Institute of Standards and Science (KRISS), Daejeon 305-340, Korea. ${ }^{7}$ Department of Materials Science and Engineering, Hanbat National University, Deajeon 34014, Korea. ${ }^{8}$ Department of Materials Science and Engineering, Pohang University of Science and Technology (POSTECH), Pohang 790-784, Korea. ${ }^{\star}$ These authors contributed equally to this work. Correspondence and requests for materials should be addressed to M.-J.L. (email: myoungjae.lee@dgist.ac.kr) or M.-H.J. (email: mhjo@postech.ac.kr).
} 
W aste heat has been the most significant loss of useful energy in heat engines since their discovery. Recovery of waste heat will enhance energy efficiency, reduce greenhouse emission and promote sustainable development. Thermoelectric (TE) devices have shown potential for reclaiming waste heat, whereas TE generators contain no mechanical parts and are therefore useful for long-term operation.

TE effect refers to a phenomena wherein a temperature difference across a material creates an electric potential, commonly called the Seebeck effect ${ }^{1-5}$, or the opposite case where electric potential creates a temperature difference, as seen in Peltier cells ${ }^{1,6}$. Since its discovery, researchers have struggled to improve the efficiency of the TE, represented by the dimensionless figure of merit, $Z T=\left(S^{2} \sigma / \kappa\right) T$, where $S, \sigma$ and $\kappa$ are the Seebeck coefficient (also called TE power), the electrical conductivity and the thermal conductivity of the material, respectively. To improve $Z T$ we have continued to design materials and structures that have an enhanced electrical conductivity $\sigma$ and reduced thermal conductivity $\kappa$. Recent research into nanomaterials has led to increased $Z T$ through nanomaterials or -structures and quantum confinement effects to obtain an enhanced density of states near the Fermi energy ${ }^{2-11}$.

As nanoscale structures have become known, their counterintuitive properties have intrigued experimental and theoretical workers. The benefits of nanoscale structures have been applied across various fields from fin-type field-effect transistor structures for semiconductors ${ }^{12}$ to drug delivery ${ }^{13}$ and targeting in medicine ${ }^{14}$. In view of TE materials, several different types of nanostructures such as $\mathrm{Si}$ nanowires $\left(Z T_{200 \mathrm{~K}} 1\right)^{15}$, quantum

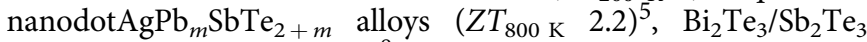
superlattices $\left(Z T_{300 \mathrm{~K}} 2.4\right)^{8}$ and layered structure of twodimensional (2D) crystalline sheets ${ }^{16,17}$ have been used to improve $Z T$ by using low-dimensional structures. These general ideas have been used to greatly decrease the thermal conductivity, while suppressing the decrease in electrical conductivity as shown in Fig. 1. The progress of TE materials are described in Fig. 1, showing the trend of $Z T$ for different materials classes.

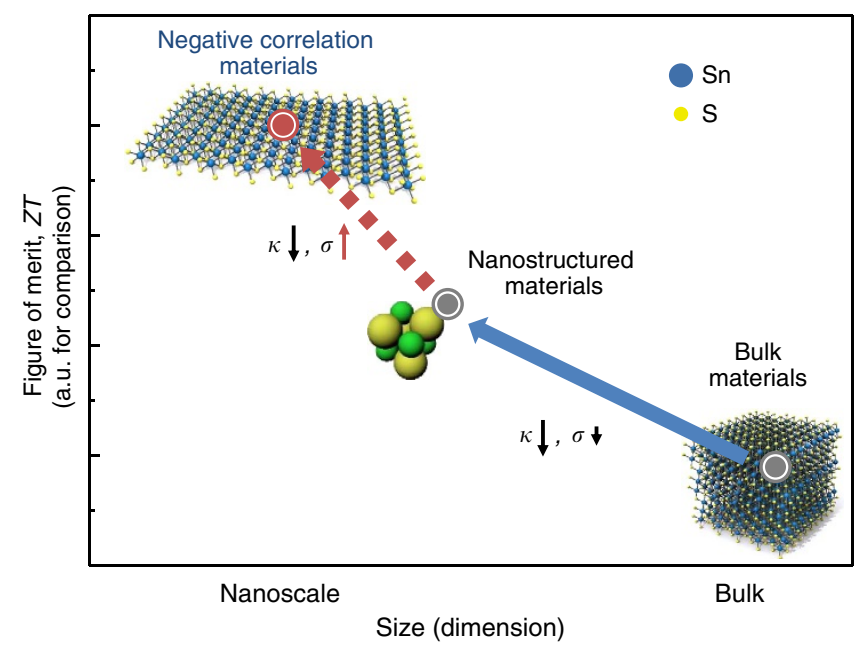

Figure 1 | Pathway and progress for high ZT in TE materials.

Conventional bulk materials have values from 0.01 to around 1 , by using nanostructures, values between 0.1 to 2 have been reported for SnSe $\left(Z T_{300}\right.$ k 0.12; ref. 4), quantum nanodot $\mathrm{AgPb}_{m} \mathrm{SbTe}_{2+m}$ alloys $\left(Z T_{800 \mathrm{~K}} 2.2\right.$; ref. 5$), \mathrm{Bi}_{2} \mathrm{Te}_{3} / \mathrm{Sb}_{2} \mathrm{Te}_{3}$ superlattices $\left(Z \mathrm{~T}_{300 \mathrm{~K}} 2.4 ;\right.$ ref. 8 ) and Si nanowires $\left(Z T_{200 \mathrm{k}} 1\right.$; ref. 15$)$. We present negatively correlated materials, which offer another method for increase of $Z T$. The solid blue arrow indicates previous progress, while the dotted red arrow is our proposed approach for further increased $Z T$.
More recently, with the discovery of the 2D carbon allotrope graphene $^{18}$, a great deal of interest has been focused into $2 \mathrm{D}$ nanostructures ${ }^{16,17}$. Most importantly for TE materials, the electrical conductivity of layered 2D materials were in some cases greater than their bulk material counterparts ${ }^{18}$. Surprisingly, for nanoscale $\mathrm{SnS}_{2}$ we found that although the electrical conductivity increases in $2 \mathrm{D}$ structures, the thermal conductivity in fact decreases. This class of materials leads to negative correlation between enhanced electrical conductivity $\sigma$ and reduced $\kappa$ exactly as we would like for TE applications. $\mathrm{SnS}_{2}$ has a unique structural property of layered $\mathrm{CdI}_{2}$-type structure; the tin ( $\mathrm{Sn}$ ) atoms are sandwiched by two layers of hexagonally packed sulfide $(\mathrm{S})$ atoms ${ }^{19}$. Each layer has a thickness of 6-7 $\mathrm{A}$. The intra-layer metal (M)-chalcogenide (X) bonds are predominantly covalent in nature, whereas the layers themselves are coupled by weak van der Waals ${ }^{19}$ bonds. The metal atoms provide four electrons to fill the bonding states of $\mathrm{SnS}_{2}$ such that the oxidation states of the $\mathrm{M}$ and $\mathrm{X}$ atoms are +4 and -2 , respectively. The lone-pair electrons of the chalcogen atoms terminate the surfaces of the layers and the absence of dangling bonds renders those layers stable against reactions with environmental species. In addition, very recent theoretical work using first principle calculations in ref. 20 have suggested $Z T$ values as high as 0.96 at room temperature, which shows potential for TE applications.

\section{Results}

Synthesis and investigations of TE properties of $\mathrm{SnS}_{2}$. We were able to succeesfully fabricate pure $\mathrm{SnS}_{2}$ single crystals by thermal chemical vapour transport (CVT). To our knowledge, this is first time CVT has been successfully used to fabricate $\mathrm{SnS}_{2}$ single crystals for electrical measurement (for further growth details and characterization of $\mathrm{SnS}_{2}$ nanosheets, see Methods and Supplementary Figs $1-4$ ).

One method of investigating the TE effect in materials has been the use of focused laser to generate thermal current through local heating, creating a temperature gradient ${ }^{16,17}$. We investigated the total photocurrent in 16-nm-thick $\mathrm{SnS}_{2}$ sample by using laser wavelengths of $405 \mathrm{~nm}(3.06 \mathrm{eV})$. The beam spot size was $500 \mathrm{~nm}$ and scanned across the entire crystal, while measuring the current through grounded drain electrodes. The experimental setup and explanations are included in Fig. 2a. Figure $2 \mathrm{~b}$ shows the photocurrent map ( $0 \mathrm{~V}$ source bias) under the laser power of $45 \mu \mathrm{W}$. The large current values appearing on the source/drain electrodes indicate the presence of high thermal current; in contrast, most materials show only current at the metal/semiconductor interface due to photoelectric currents ${ }^{17}$.

The thickness of our samples was measured by atomic force microscopy (AFM) and confirmed to be $16 \mathrm{~nm}$ as shown in Fig. $2 \mathrm{c}, \mathrm{d}$. Figure $2 \mathrm{e}$ shows the reflection image of Fig. $2 \mathrm{~b}$ and numerical derivative (Fig. $2 \mathrm{f}$ ) of the reflection signal, which allows for a clear indication of source/drain and $\mathrm{SnS}_{2}$ boundaries. Figure $2 \mathrm{~g}$ shows ultraviolet-visible absorption spectroscopy. The absorption edge of $\mathrm{SnS}_{2}$ films for a 16-nm-sample show a bandgap $\left(E_{\mathrm{g}}\right)$ of $2.59 \mathrm{eV}$. Meanwhile, the $100-\mathrm{nm}$-thick $\mathrm{SnS}_{2}$ film was measured to have a bandgap of $2.15 \mathrm{eV}$ (for the dependence of optical bandgap on $\mathrm{SnS}_{2}$ thickness, see Supplementary Fig. 5).

We also investigated the photocurrent in two different $\mathrm{SnS}_{2}$ samples of thickness 16 and $100 \mathrm{~nm}$ in the same way as previously shown in Fig. 2a. Figure 3a shows the photocurrent and reflectance images at $0 \mathrm{~V}$ source bias and Fig. $3 \mathrm{~b}$ shows photocurrent profile of a 100 -nm-thick $\mathrm{SnS}_{2}$ crystal illuminated with laser power of $195 \mu \mathrm{W}$ laser. The large Gaussian current profile is not due to the photovoltaic effect, but a photo-TE effect, similar to previous reports ${ }^{17}$. However, we should note that the 
a

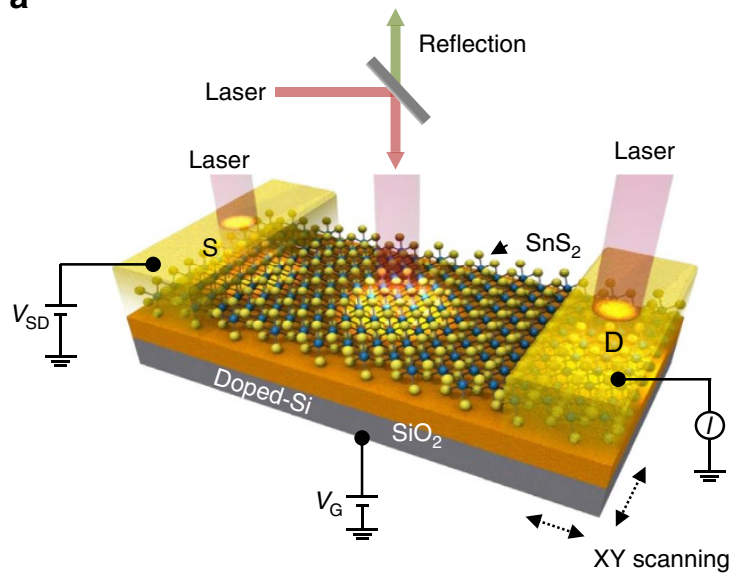

b

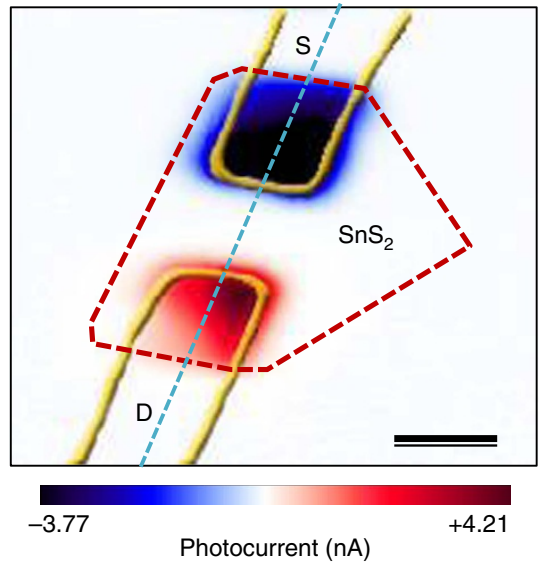

C

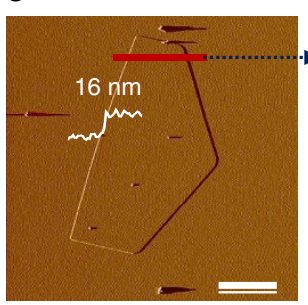

d

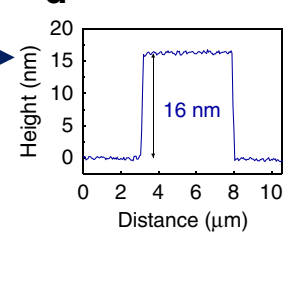

e

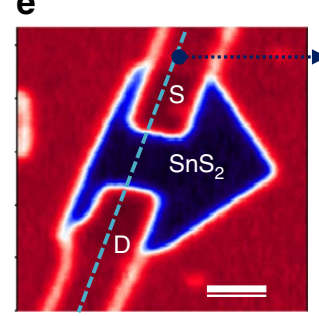

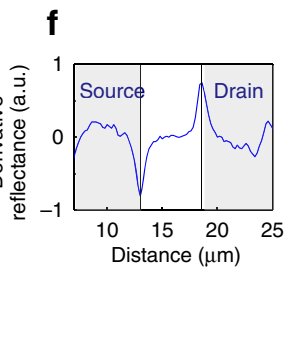

g

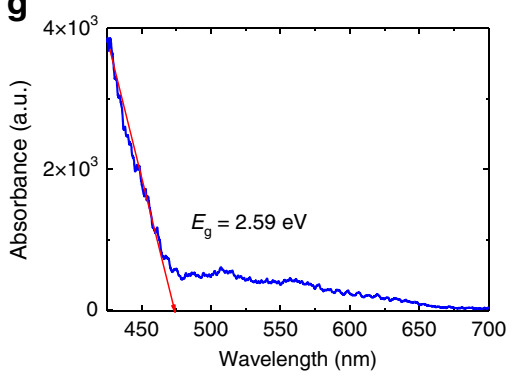

Figure 2 | Thermal current measurement of $\mathbf{S n S}_{\mathbf{2}}$ by laser scanning photoinduced TE current imaging. Devices are $16 \mathrm{~nm}$ thick. (a) Schematic of the photo-TE measurement setup with a laser wavelength of $\lambda=405 \mathrm{~nm}$ and laser power of $45 \mu \mathrm{W}$, where we simultaneously collect images of photo-TE current and optical reflectance as a function of the laser position. (b) Scanning photo-TE current imaging ( $\mathrm{V}$ source/drain bias), which demonstrates largely thermocurrent-dominated profile in the source (S)/drain (D) electrode regions. The outline of the electrodes (yellow solid lines) and the SnS 2 nanosheet (red dashed line) are indicated from reflection image of e. Scale bar, $3 \mu \mathrm{m}$. (c) AFM image of SnS 2 . Scale bar, $5 \mu \mathrm{m}$. (d) Thickness measurement along the red line in the AFM image. (e) Reflection image. Scale bar, $3 \mu \mathrm{m}$. (f) Numerical derivative of the reflectance data along the blue dashed line allows for clear indication of S/D and $\mathrm{SnS}_{2}$ boundaries. (g) Ultraviolet-visible absorption spectroscopy. The absorption edge of $\mathrm{SnS}_{2}$ films showing the bandgap at $2.59 \mathrm{eV}$, extrapolated from the $x$ intercept of the linear portion of our data (red line).

larger Gaussian current profile for $100 \mathrm{~nm}$ samples compared with $16 \mathrm{~nm}$ ones is probably due to the lowering of the bandgap, leading to a more pronounced photovoltaic effect in the thicker samples as shown in Supplementary Fig. 5. We also used a $532-\mathrm{nm}(2.33 \mathrm{eV})$ laser on the same $100-\mathrm{nm}$ sample and found that the current features are similar even though $h v<E_{\mathrm{g}}$ of $\mathrm{SnS}_{2}$ (see Supplementary Fig. 6). To clearly identity source/drain interfaces, we used the measured reflectance (right of Fig. 3a) and show the derivative (blue dotted line of fig. 3b). The local minimum and maximum of the blue dotted line indicate the position of the source/drain interfaces. The solid red and blue lines in the photocurrent profiles of Fig. 3b,c are the Gaussian components and the results after subtracting each Gaussian component from the experimental data, respectively.

Figure $3 \mathrm{c}$ shows photocurrent profile ( $0 \mathrm{~V}$ source/drain bias) of a $16-\mathrm{nm}$-thick $\mathrm{SnS}_{2}$ (2D layered structure) crystal illuminated with laser power of $45 \mu \mathrm{W}$. The photocurrent profile is extracted from the photocurrent image previously shown in Fig. 2b. The photo-TE profile was changed compared with the 100-nm sample as observed in Fig. 3b. Figure $3 \mathrm{~d}$ shows current-voltage $(I-V)$ characteristics (source-drain) of $\mathrm{Ti} / \mathrm{Au}$ contact on $\mathrm{SnS}_{2}$ nanosheet exhibited ohmic behaviour. If the photocurrent is dominated by photo-TE current, we can expect a current profile as the one in the diagram of Fig. 3e. Here we notice a Gaussian profile reflecting the intensity profile of the laser beam spot. The Gaussian peak is centred at the metal-semiconductor interface where the internal field due to the Schottky barrier is maximized.
Almost identical profiles have been previously reported for $\mathrm{MoS}_{2}$ materials where photo-TE effects dominate the photocurrent ${ }^{17}$. When the TE effect dominates the photocurrent, we expect a different distribution rather than the Gaussian profile, because the temperature gradient rather than the laser intensity affects carrier distribution. The distribution peak is also moved into the electrodes, rather than the interface, as explained by the diagram of Fig. 3f. These features are well in agreement with our observed photocurrent profiles.

The layered structure materials including $\mathrm{SnS}_{2}$ can be cleaved down to few or single layer, with significant changes to the electrical and optical properties such as indirect-to-direct bandgap transition ${ }^{21,22}$. The evolution of electronic properties of $\mathrm{SnS}_{2}$ nanosheets with various thicknesses can be reflected in their photo or thermal current. The bulk $\mathrm{SnS}_{2}$ showed mall thermal current, whereas thinner $\mathrm{SnS}_{2}$ nanosheets exhibited pronounced thermal current at $16 \mathrm{~nm}$ thickness (Figs $2 \mathrm{~b}$ and $3 \mathrm{~b}$ ).

Negative correlation between electrical and thermal conductivity. Our results suggest that the TE effect is the dominant photocurrent mechanism in layered $2 \mathrm{D}(16 \mathrm{~nm}) \mathrm{SnS}_{2}$. We believe this is due to the unique properties of $2 \mathrm{D} \mathrm{SnS}_{2}$ where the thermal conductivity decreases with decreasing thickness, due to surface phonon scattering, whereas at the same time electrical conductivity increases with decreasing thickness, possibly due to changes in the band structure similar to graphene ${ }^{18}$. By using 
a
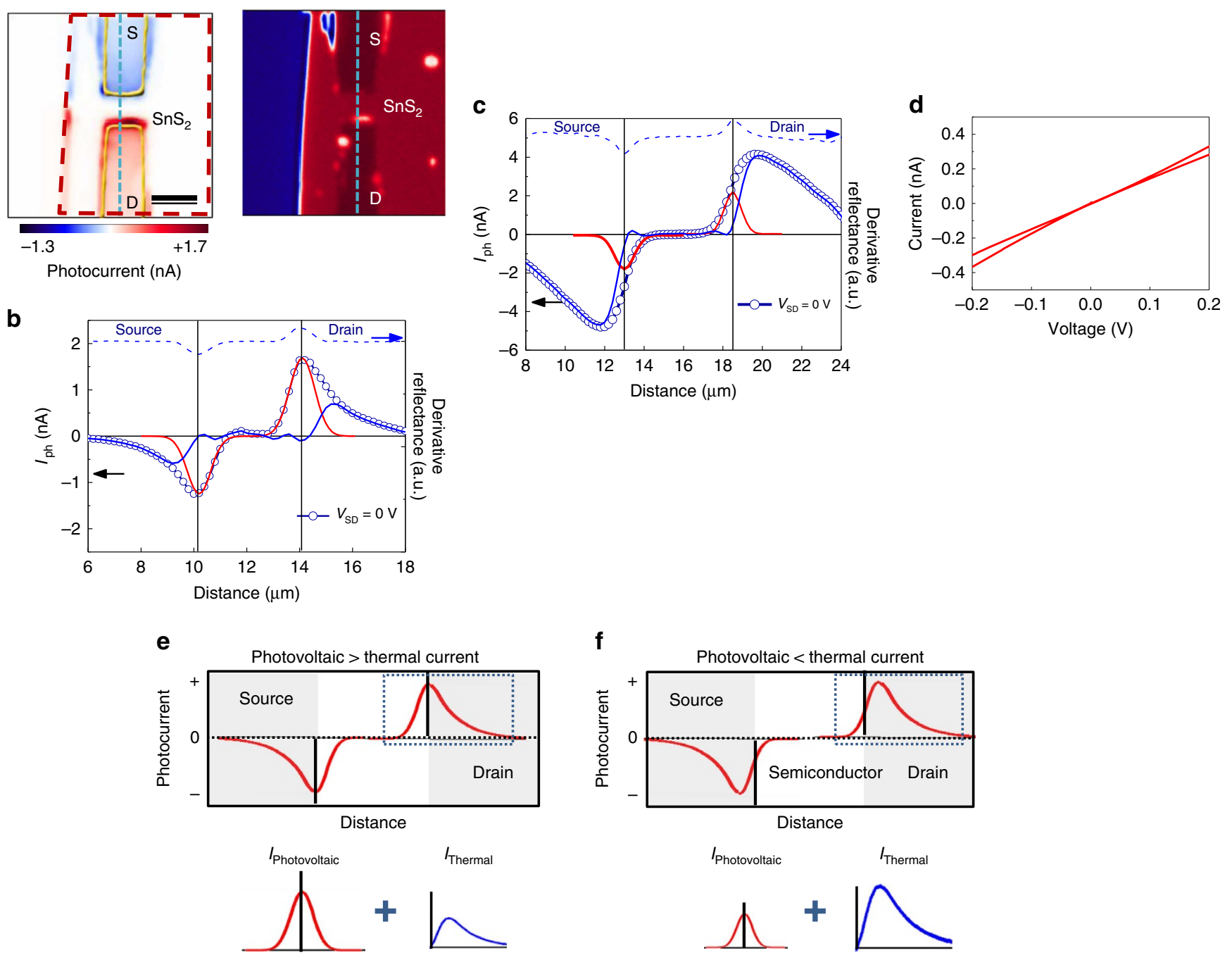

Figure 3 | Photocurrent composition measurement and analysis for two $\mathbf{S n S}_{\mathbf{2}}$ samples of thickness. (a) Photocurrent map (left) and reflection image (right). Scale bar, $3 \mu \mathrm{m}$. (b) Photocurrent profile at $0 \mathrm{~V}$ source bias with laser wavelength of $405 \mathrm{~nm}(3.06 \mathrm{eV}$ ) and power of $195 \mu \mathrm{W}$ for $100 \mathrm{~nm}$ thickness, which shows photovoltaic current-dominated Gaussian profile. The derivative of reflectance along the blue dashed line shown in the reflectance measurements (right of a) plotted in the photocurrent profile. (c) Photo-TE current profile from along the blue dashed line in Fig. 2b, which demonstrates a thermocurrent-dominated profile. (d) I-V curve of the Pt/Ti/SnS 2 contact, demonstrating that a good ohmic contact is formed on the $\mathrm{SnS}_{2}$ material. (e,f) Expected current profile and decomposition of contributing components for the 100- (e) and 16-nm (f) samples. Shaded areas indicate S/D electrode regions. The measured photocurrent in the dotted blue rectangles of $\mathbf{e}$ (top) and $\mathbf{f}$ (top) are decomposed into their respective Gaussian photovoltaic (bottom left) and thermal (bottom right) components; their sum results in the profile shown in each respective dotted blue rectangle region.

this property it may be possible to maximize $Z T$ beyond any previous approach. We have done an electron density change calculation by using Synopsys technology computer-aided design for $\mathrm{SnS}_{2}$, showing increased electron density at reduced thickness, which we present in Supplementary Fig. 7, Supplementary Note 1 and Methods.

To further investigate the changes to thermal conductivity in $2 \mathrm{D} \mathrm{SnS}_{2}$ crystal, we use the previously reported microfabricated TE measurement platform (MTMP) device, which was shown as a method to accurately measure $\mathrm{Bi}_{2} \mathrm{Te}_{3}$ thin film conductivities previously $^{23}$. In brief, the MTMP method uses a differential method to exactly measure thermal conductivity of materials precisely by using a microelectromechanical system-based device $^{15,23}$. A full description of the MTMP method is included in the Supplementary Figs 8 and 9. The results of the 16-nm film show a thermal conductivity of $3.45 \mathrm{~W} \mathrm{~m}^{-1} \mathrm{~K}^{-1}$ at room temperature; the temperature-dependent thermal conductivity is shown in Fig. 4a and plotted alongside is the reported value for bulk $\mathrm{SnS}_{2}$ in ref. 24 at $300 \mathrm{~K}$. A separate $22-\mathrm{nm}$ sample was also measured later, to confirm the thermal conductivity dependence (Supplementary Fig. 10), and included in Fig. 4a. In comparison with bulk, this is around one-third of the reported $10 \mathrm{~W} \mathrm{~m}^{-1} \mathrm{~K}^{-1}$ in ref. 24 at $300 \mathrm{~K}$. Figure $4 \mathrm{~b}$ shows scanning electron microscopy image of MTMP structure used for thermal conductivity measurements with current-supplying nanoheater and temperature-measuring thermometer metal leads. The sheet resistivity of $\mathrm{SnS}_{2}$ nanosheets was measured using a four-point Van-der Pauw method for samples from 120 to $3 \mathrm{~nm}$ thickness (for four-point Van-der Pauw method, see Supplementary Fig. 11). We multiplied the film thickness confirmed by AFM and took the inverse value to find electrical conductivity $\sigma(T)$ in Fig. 4c. Between 16 and $50 \mathrm{~nm}$ of $\mathrm{SnS}_{2}$ thickness, there is a 30 times increase in the electrical conductivity from $\sim 4 \times 10^{-4}$ to $\sim 1 \times 10^{-2} \mathrm{Scm}^{-1}$. We believe this region to describe the 
a

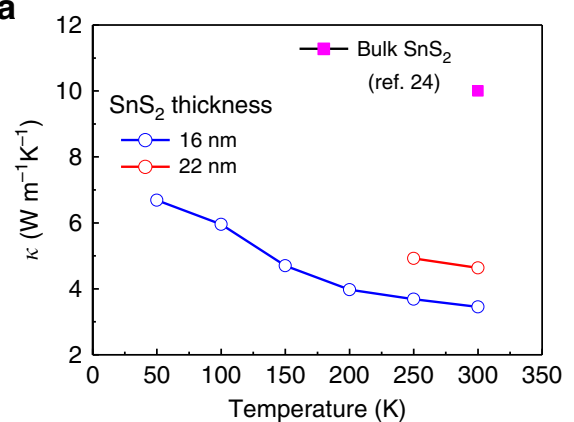

d

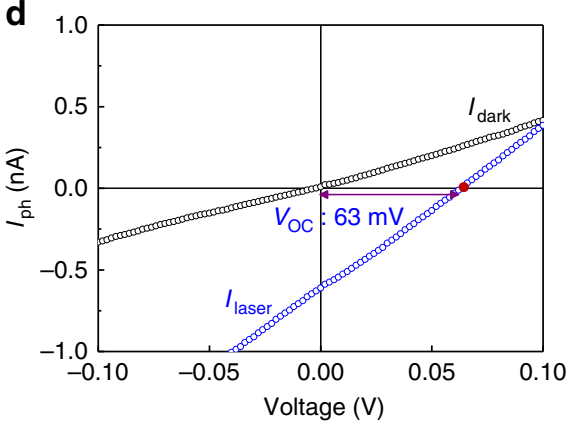

b

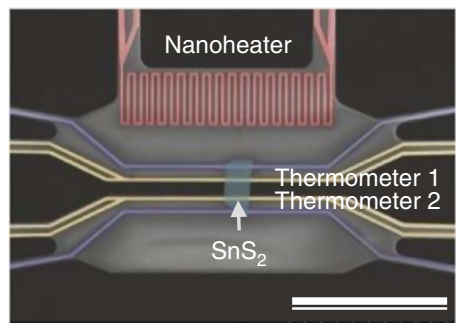

C

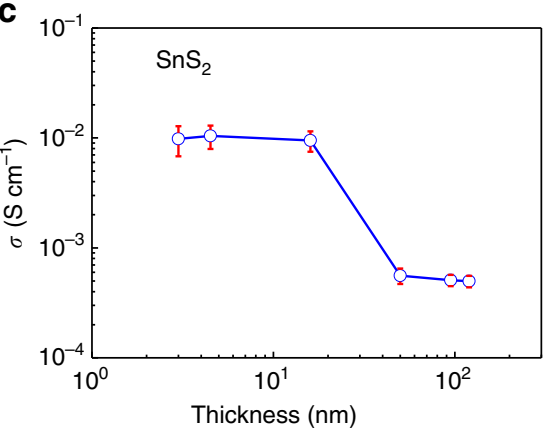

f

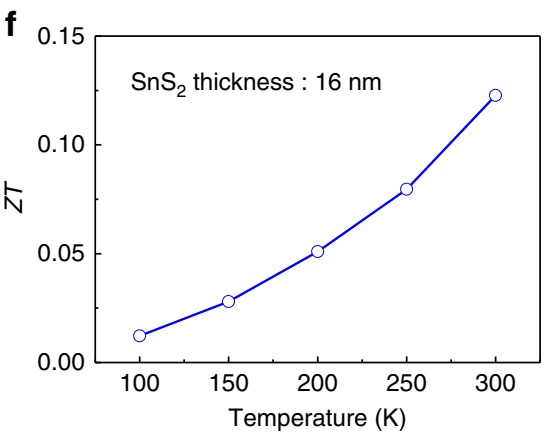

Figure 4 | Negatively correlated electrical and thermal conductivity. (a) Thermal conductivity measurements for 16- and 22-nm-thick $\mathrm{SnS} \mathrm{S}_{2}$ samples compared with bulk $\mathrm{SnS}_{2}$ reported values. (b) Scanning electron microscopy image used for MTMP setup and precise thermal conductivity measurement. Scale bar, $30 \mu \mathrm{m}$. (c) Electrical conductivity measurements for different thickness of $\mathrm{SnS}_{2}$ showing a large increase below $16 \mathrm{~nm}$. The median value of three samples is shown by open circles, whereas error bars indicate the maximum and minimum values from each sample. (d) Open circuit voltage $\left(V_{o c}=63 \mathrm{mV}\right)$ measurement of $16-\mathrm{nm} \mathrm{SnS}_{2}$ sample under illumination by $405-\mathrm{nm}$ laser (power of $45 \mu \mathrm{W}$ ). (e) Simulated $\Delta T$ for SnS 2 samples illuminated by the same laser as left-hand side figure. (f) Calculated ZT values for temperatures from 100 to $300 \mathrm{~K}$.

change from bulk three-dimensional properties to $2 \mathrm{D}$ properties for $\mathrm{SnS}_{2}$ thin films. Meanwhile, for bulk $\mathrm{SnS}_{2}$ electrical conductivity has been reported as $0.9 \mathrm{~S} \mathrm{~cm}^{-1}$ (ref. 25).

\section{Discussion}

From $Z T=\left(S^{2} \sigma / \kappa\right) T$, everything else being constant, the one-third decrease in $\kappa$ and the 30 times increase in $\sigma$ lead to a total ZT increase of over 90 times for $2 \mathrm{D} \mathrm{SnS}_{2}$ in comparison with that of three-dimensional $\mathrm{SnS}_{2}$. Finally, the Seebeck coefficient was measured by using the open circuit voltage $\left(V_{\text {oc }}\right)$ of devices as shown in Fig. 4d, leading to $V_{\mathrm{oc}}=63 \mathrm{mV}$ for $16 \mathrm{~nm}$ devices (for the Seebeck coefficient for $150-\mathrm{nm} \mathrm{SnS}_{2}$ thickness, see Supplementary Fig. 12).

Using the definition of the Seebeck coefficient and simulated $\Delta T$ value of $1.667 \mathrm{~K}$ (Fig. 4e) for conditions exactly as described in Fig. 2b, we have $|S|=26.1-34.7 \mathrm{mV} \mathrm{K}^{-1}$ ( $\Delta T$ simulations along with calibration methods using $\mathrm{Bi}_{2} \mathrm{Te}_{3}$ described in Supplementary Figs 13 and 14, Supplementary Notes 2 and 3, and Supplementary Table 1), with an overestimation error of $\sim 28 \%$. In comparison, the reported absolute values for bulk $\mathrm{Sn}_{x} \mathrm{~S}_{y}$ are $0.1-4.6 \mathrm{mV} \mathrm{K}^{-1}$ in ref. 26 and measurement values for our 100 -nm-thick $\mathrm{SnS}_{2}$ sample are around $3.2 \mathrm{mVK}^{-1}$, with an overestimation error of $\sim 28 \%$. We expect a large increase in $S$ to enhance local electron density from confinement effects, similar to previously reported nanostructures ${ }^{6}$. Finally, we calculate $Z T$ values $\left(16 \mathrm{~nm} \mathrm{SnS}_{2}\right)$ from 0.012 at $100 \mathrm{~K}$ to 0.13 at $300 \mathrm{~K}$ from our measured values as shown in Fig. $4 \mathrm{f}$, which are close to the highest values being reported for $\mathrm{TE}$ materials currently $^{3-6}$. The measured electrical conductivity dependence on temperature for 16 and $100-\mathrm{nm}$ samples is included in the Supplementary Fig. 15.
In summary, at this time the final $Z T$ values was around other reported nanomaterial values of 0.13 at $300 \mathrm{~K}$. We believe a significant increases can be made by theoretical screening for materials, which have negatively correlated electrical and thermal conductivity with a layered structure similar to $\mathrm{SnS}_{2}$, while having higher absolute electrical conductivity. Currently, researchers believe a room temperature $Z T$ of 0.5 (a factor of 4 higher than this work), which could reach a $Z T$ of over 3 at high temperature $(900 \mathrm{~K})$, to be useful for industrial applications ${ }^{3,4}$. By investigating materials that have negative correlation between $\sigma$ and $\kappa$, we suggest that moderate-temperature TE materials can be discovered.

\section{Methods}

Synthesis and characterization of $\mathbf{S n S}_{\mathbf{2}}$ nanosheets. Synthesis of $\mathrm{SnS}_{2}$ was carried out by thermal CVT in an evacuated tube furnace using 300-nm-thick $\mathrm{SiO}_{2}$ substrate. $\mathrm{SnO}_{2}(99 \%$, nanopowder with particle size under $100 \mathrm{~nm}$, SigmaAldrich) and sulfur powder ( $99 \%$, Sigma-Aldrich) precursors were used for the synthesis of $\mathrm{SnS}_{2}$, with temperature range of $600-680^{\circ} \mathrm{C}$ (Supplementary Fig. 1). Triangular faceting of single-crystal $\mathrm{SnS}_{2}$ was observed by optical microscopy. The crystal structure and their characterization was confirmed with transmission electron microscopy, Raman and absorption spectrum (see Supplementary Figs 2 and 3). The morphology and the number of layers are determined by Raman and AFM imaging.

Electrical characterization of $\mathbf{S n S}_{\mathbf{2}}$. $\mathrm{SnS}_{2}$ samples for laser and electrical measurement were fabricated by electron-beam lithography on the wafers that were previously doubly spun with two polymethyl methacrylate layers. Electrodes are evaporated in a high vacuum e-beam evaporator and are composed of $5 \mathrm{~nm}$ per $50 \mathrm{~nm}$ titanium (Ti)/gold ( $\mathrm{Au}$ ) layers. Subsequently, the polymethyl methacrylate/ $\mathrm{Ti} / \mathrm{Au}$ layer is lifted off in acetone. The basic electronic transport characterization of the $\mathrm{SnS}_{2}$ devices is performed at room temperature and atmosphere. The scanning photocurrent microscopy is carried out at room temperature in a confocal microscope setup with an objective with numerical aperture $=0.8$. The excitation was provided by a focused laser of a given wavelength $(405$ and $532 \mathrm{~nm})$ and by a supercontinuum white-light source (Fianium Ltd) combined with a 


\section{Table 1 | The parameters of $\mathrm{SnS}_{\mathbf{2}}$ for the device simulation}

\section{Parameter}

Value

\section{Band gap}

Dielectric constant

Electron affinity

Effective mass of electron

Doping density of $\mathrm{SnS}_{2}$

Interface trap density

Energy level of interface trap

Electron mobility

monochromator for the high-resolution spectra $(450 \mathrm{~nm} \leq \lambda \leq 2,000 \mathrm{~nm})$ where $\lambda$ is the wavelength. During the wavelength scanning, photocurrent is measured by a lock-in technique with the chopper frequency of $500 \mathrm{~Hz}$ and subsequently normalized to the photon flux. The chopped laser beam is focused by microscopic lens (numerical aperture $=0.8$ ) and illuminates the $\mathrm{SnS}_{2}$ channel and $\mathrm{S} / \mathrm{D}$ electrode region of devices.

\section{Fabrication and measurement for the thermal conductivity. The thermal} conductivity of $\mathrm{SnS}_{2}$ nanosheets was measured by using the MTMP based on the differential method first demonstrated in ref. 23 . That is, to exactly measure the thermal conductivity, we used the difference of the heat flow between devices including $\mathrm{SnS}_{2}$ flakes and without $\mathrm{SnS}_{2}$ flakes (see Supplementary Figs $8-10$ ). MTMP structures with current supplying and temperature measuring metal leads were fabricated on $\mathrm{Si}_{3} \mathrm{~N}_{4}\left(50 \mathrm{~nm}\right.$ thick)/300-nm-thick $\mathrm{SiO}_{2}$ substrates. The microsized electrode patterns ( $\mathrm{Pt}$ nanoheater, current-supplying electrodes (outer electrodes) and Pt thermometers (inner electrodes)) were photolithographically defined as shown in Supplementary Fig. 8. For enhancing the measurement sensitivity, the device area between the inner electrodes was removed: first, by etching the $\mathrm{Si}_{3} \mathrm{~N}_{4} / \mathrm{SiO}_{2}$ wafer front side with hydrogen fluoride (HF) solution, then aligning the backside photoresist patterns to expose the $\mathrm{Si}$ and etching with a $30 \% \mathrm{KOH}$ etching solution at $353 \mathrm{~K}$. The temperatures were obtained from the resultant responses of the Pt thermometers to electrical resistance variations. The temperature gradient, $\Delta T$, was generated by the Joule heating using a DC current (Keithley, 6220) in the range $0-64 \mu \mathrm{A}$ through the Pt nanoheater. Two lock-in amplifiers (Signal Recovery, 5210) simultaneously read the resistances of both thermometers. The resistances of the Pt thermometers were converted into temperature values using the temperature coefficient of resistance (TCR). We determined the TCR for each individual microelectromechanical systems device before conducting the TE measurement, as a small difference in the TCR would lead to significant deviation from the real value when reading the temperature. From 200 to $400 \mathrm{~K}$, the resistances of the Pt thermometers, which changed linearly with the temperature, were measured at the interval of $20 \mathrm{~K}$ in the temperature range of $200-400 \mathrm{~K}$. The TCRs were determined from the slope of resistance versus temperature.

Calculation methods. Electron density profiles and normalized conductance data shown in Supplementary Fig. 7b,c are calculated by Synopsys technology computer-aided design, a commercial semiconductor device simulator in ref. 27. Poisson equations and drift-diffusion equations are selected for calculating electrostatic potential and carrier transport, respectively, which are represented by following equations:

$$
\begin{aligned}
\nabla \cdot(\varepsilon \nabla \psi) & =-q\left(p-n+N_{\mathrm{D}}-N_{\mathrm{A}}\right) \\
\mathbf{J}_{n} & =-n q \mu_{n} \nabla \Phi_{n} \\
\mathbf{J}_{p} & =-p q \mu_{p} \nabla \Phi_{p}
\end{aligned}
$$

where $\varepsilon, \psi$ and $q$ are the electrical permittivity, the electrostatic potential and the elementary electronic charge, respectively; $n$ and $p$ are the electron and the hole densities, $N_{\mathrm{D}}$ and $N_{\mathrm{A}}$ are the concentration of ionized donors and acceptors, respectively; $\Phi_{n}=-E_{\mathrm{F}_{\mathrm{n}}} / q$ and $\Phi_{p}=-E_{\mathrm{F}_{\mathrm{p}}} / q$ are the quasi-Fermi potentials for the electrons and holes, respectively; $\mathbf{J}_{n}$ and $\mathbf{J}_{p}$ are the electron current density and the hole current density, respectively. The generation of $n$-doped region by surface states of $\mathrm{SnS}_{2}$ is modelled by shallow donor-like interface trap formation. The schematic and the parameters for the device simulation are described in the Supplementary Fig. 7a and Table 1, respectively.

Data availability. The data that support the findings of this study are available from the corresponding author upon request.

\section{References}

1. Tritt, T. M. et al. Thermoelectric materials, phenomena, and applications: A bird's eye view. Mater. Res. Soc. Bull. 31, 188-198 (2006).
2. Hicks, L. D. \& Dresselhaus, M. S. Effect of quantum-well structure on the thermoelectric figure of merit. Phys. Rev. B 47, 12727-12731 (1993).

3. Rhyee, J.-S. et al. Peierls distortion as a route to high thermoelectric performance InSe crystals. Nature 459, 965-968 (2009).

4. Zhao, L.-D. et al. Ultralow thermal conductivity and high thermoelectric figure of merit in SnSe crystals. Nature 508, 373-377 (2014).

5. Harman, T. C., Taylor, P. J., Walsh, M. P. \& LaForge, B. E. Quantum dot superlattice thermoelectric materials and devices. Science 297, 2229-2232 (2002).

6. Harman, T. C., Cahn, J. H. \& Logan, M. J. Measurement of thermal conductivity by utilization of the Peltier effect. J. Appl. Phys. 30, 1351-1359 (1959).

7. Ohta, H. et al. Giant thermoelectric Seebeck coefficient of a two-dimensional electron gas in $\mathrm{SrTiO}_{3}$. Nat. Mater. 6, 129-134 (2007).

8. Venkatasubramanian, R., Siivola, E., Colpitts, T. \& O’Quinn, B. Thin-film thermoelectric devices with high room-temperature figures of merit. Nature 413, 597-602 (2001).

9. Poudel, B. et al. High-thermoelectric performance of nanostructured bismuth antimony telluride bulk alloys. Science 320, 634-638 (2008).

10. Bell, L. E. Cooling heating, generating power, and recovering waste heat with thermoelectric systems. Science 321, 1457-1461 (2008).

11. Snyder, G. J. \& Toberer, E. S. Complex thermoelectric materials. Nat. Mater. 7, 105-114 (2008).

12. Hisamoto, D. et al. FinFET-a self-aligned double-gate MOSFET scalable to 20 nm. IEEE Trans. Electron Devices 47, 2320-2325 (2000).

13. LaVan, D. A., McGuire, T. \& Langer, R. Small-scale systems for in vivo drug delivery. Nat. Biotechnol. 21, 1184-1191 (2003).

14. Veiseh, O., Gunn, J. W. \& Zhang, M. Design and fabrication of magnetic nanoparticles for targeted drug delivery and imaging. Adv. Drug Deliv. Rev. 62, 284-304 (2010).

15. Boukai, A. I. et al. Silicon nanowires as efficient thermoelectric materials. Nature 451, 168-171 (2008).

16. Sung, J. H. et al. Atomic layer-by-layer thermoelectric conversion in topological insulator bismuth/antimony tellurides. Nano Lett. 14, 4030-4035 (2014).

17. Buscema, M. et al. Large and tunable photothermoelectric effect in single-layer $\mathrm{MoS}_{2}$. Nano Lett. 13, 358-363 (2013).

18. Novoselov, K. S. et al. Two-dimensional gas of massless Dirac fermions in graphene. Nature 438, 197-200 (2005).

19. Zhong, H. et al. Vertically aligned Graphene-like $\mathrm{SnS}_{2}$ ultrathin nanosheet arrays: excellent energy storage, catalysis, photoconduction, and field-emitting performances. J. Phys. Chem. C 116, 9319-9326 (2012).

20. Sun, B. -Z., Ma, Z., He, C. \& Wu, K. Anisotropic thermoelectric properties of layered compounds in $\mathrm{SnX}_{2}(\mathrm{X}=\mathrm{S}, \mathrm{Se})$ : a promising thermoelectric material. Phys. Chem. Chem. Phys. 17, 29844 (2015).

21. Lokhande, C. D. A chemical method for tin disulphide thin film deposition. J. Phys. D Appl. Phys. 23, 1703-1705 (1990).

22. Chhowalla1, M. et al. The chemistry of two-dimensional layered transition metal dichalcogenide nanosheets. Nat. Chem. 5, 263-275 (2013).

23. Shin, H. S. et al. Twin-driven thermoelectric figure-of-merit enhancement of $\mathrm{Bi}_{2} \mathrm{Te}_{3}$ nanowites. Nanoscale 6, 6158-6165 (2014).

24. Khélia, C. et al. Structural, optical and thermal properties of $\beta-\mathrm{SnS}_{2}$ thin films prepared by the spray pyrolysis. Eur. Phys. J. Appl. Phys. 9, 187-193 (2000).

25. Madelung, O. Semiconductors: Data Handbook 3rd edn (Springer, 2004).

26. Fadavieslam, M. R., Shahtahmasebi, N., Rezaee-Roknabadi, M. \& BagheriMohagheghi, M. M. Effect of deposition conditions on the physical properties of $\mathrm{Sn}_{\mathrm{x}} \mathrm{S}_{\mathrm{y}}$ thin films prepared by the spray pyrolysis technique. J. Semicond. 32, $113002(2011)$

27. TCAD Sentaurus Device User's Manual (version I-2013.12), Synopsys, Mountain View, CA (2013) < https://solvnet.synopsys.com/Install/ installation_guide.jsp? $i d=146 \&$ releasedate $=2014-03-10>$.

28. Burton, L. A. et al. Synthesis, characterization, and electronic structure of single-crystal $\mathrm{SnS}, \mathrm{Sn}_{2} \mathrm{~S}_{3}$, and $\mathrm{SnS}_{2}$. Chem. Mater. 25, 4908-4916 (2013).

29. Mandalidis, S., Kalomiros, J. A., Kambas, K. \& Anagnostopoulos, A. N. Optical investigation of $\mathrm{SnS}_{2}$ single crystals. J. Mater. Sci. 31, 5975-5978 (1996).

30. Bletskan, D. I., Frolova, V. V. \& Glukhov, K. E. Electronic structure of $18 R-\mathrm{SnS}_{2}$ crystal. J. Optoelectron. Adv. Mater. 12, 2236-2241 (2010).

31. Gowers, J. P. \& Lee, P. A. Mobility of electrons in $\mathrm{SnS}_{2}$ single crystals. Solid State Conmmun. 8, 1447-1449 (1970).

\section{Acknowledgements}

This work was supported by DGIST Basic Research Program of the Ministry of Science, ICT and Future Planning (16-NB-05) and by Institute for Basic Science (IBS), Korea, under the project code of IBS-R014-G1-2016-a00. J.Y.S. and S.G.J. were supported by the KEIT funded by Ministry of Trade, Industry and Energy through the project (10050296) of 'Large scale synthesis and evaluation technology of 2-dimensional chalcogenides for next generation electronic devices'. W.L. was supported by Future-based Technology Development Program (Nano Fields) through the National Research Foundation of Korea (NRF) funded by the Ministry of Science, ICT and Future Planning (NRF-2010-0029332). We thank Dr B.H. Lee of Center for Core Research Facilities 
(CCRF) of DGIST for the support of facilities. The device simulation software (Synopsys technology computer-aided design) is supported by IC Design Education Center (IDEC).

\section{Authors contributions}

M.-J.L. and M.-H.J. designed this work and prepared the manuscript. J.-H.A. synthesized the $\mathrm{SnS}_{2}$ monolayer crystals. J.H.S. and M.-J.L. performed the photocurrent spectroscopy and analysed the data. M.-J.L., H.H., S.G.J., W.L., J.H.S. and B. C. fabricated and carried out the thermal conductivity and electrical measurements. K.-H.H. performed computational simulations. All authors discussed the results and implications, and commented on the manuscript at all stages.

\section{Additional information}

Supplementary Information accompanies this paper at http://www.nature.com/ naturecommunications
Competing financial interests: The authors declare no competing financial interests.

Reprints and permission information is available online at http://npg.nature.com/ reprintsandpermissions/

How to cite this article: Lee, M. -J. et al. Thermoelectric materials by using two-dimensional materials with negative correlation between electrical and thermal conductivity. Nat. Commun. 7:12011 doi: 10.1038/ncomms12011 (2016).

(c) (i) This work is licensed under a Creative Commons Attribution 4.0 International License. The images or other third party material in this article are included in the article's Creative Commons license, unless indicated otherwise in the credit line; if the material is not included under the Creative Commons license, users will need to obtain permission from the license holder to reproduce the material. To view a copy of this license, visit http://creativecommons.org/licenses/by/4.0/ 